\title{
Analysis of global geomagnetic variability
}

\author{
V. Anh ${ }^{1,2}$, Z.-G. Yu ${ }^{1,3}$, and J. A. Wanliss ${ }^{4}$ \\ ${ }^{1}$ School of Mathematical Sciences, Queensland University of Technology, GPO Box 2434, Brisbane, Q4001, Australia \\ ${ }^{2}$ Florida Space Institute, University of Central Florida, Orlando, Florida, 32816-2370, USA \\ ${ }^{3}$ School of Mathematics and Computational Science, Xiangtan University, Hunan, 411105, China \\ ${ }^{4}$ Embry-Riddle Aeronautical University, 600 S. Clyde Morris Blvd., Daytona Beach, Florida, 32114, USA
}

Received: 9 July 2007 - Revised: 18 October 2007 - Accepted: 18 October 2007 - Published: 23 November 2007

\begin{abstract}
The orthogonal field components from global INTERMAGNET magnetometer stations are studied via multifractal detrended fluctuation analysis to determine whether there are clear and consistent regional patterns in the behavior of the fluctuations. There are three distinct scaling regimes in the qth-order fluctuation function for each of the 24 stations studied covering Southwest North America, Northeast North America, Central Europe, Northern Europe, Australasia and Asia. There is a consistent break point at time scale around $23 \mathrm{~h}$ for all stations. The scaling exponents of the second-order fluctuation functions reflect the regional character of the stations, and can be used for station classification, and for possible regional models.
\end{abstract}

\section{Introduction}

The Earth acts like a great magnet and its magnetic fluctuations are measured almost continuously by arrays of magnetometers located around the world. The INTERMAGNET program has established a global network of cooperating digital magnetic observatories that currently comprises over 100 observatories. Typical measured parameters include the north $\left(B_{x}\right)$ and east $\left(B_{y}\right)$ components of the horizontal intensity, and the vertical intensity $\left(B_{z}\right)$, or some combination of these. Because the Earth's magnetic field is constantly changing, it is a difficult task to accurately predict what the field will be at any point in the future. An important goal of space weather studies is to develop spatiotemporal models of magnetic fluctuations with reasonable accuracy. By constantly measuring the magnetic field through programs such as INTERMAGNET, we can observe how the field is changing over a period of years and use it to derive a mathematical representation of the Earth's main magnetic field and how it

Correspondence to: $\mathrm{Z} .-\mathrm{G} . \mathrm{Yu}$

(z.yu@qut.edu.au) is changing. A statistical method accurately characterizing the data can enable identification of the underlying fundamentals and dynamics of the phenomenon under study.

In the recent literature, fractal and multifractal approaches have been quite successful in extracting salient features of physical processes responsible for the near-Earth magnetospheric phenomena (Lui, 2002). Heavy-tailed Lévy-type behaviour has been observed in the interplanetary medium and the magnetosphere (Burlaga, 1991, 2001; Burlaga et al., 2003; Kabin and Papitashvili, 1998; Lui et al., 2000, 2003). Wanliss $(2004,2005)$ and Wanliss and Dobias (2007) have found that the $D_{s t}$ index exhibits a power-law spectrum with the Hurst parameter varying over different segments of the time series. This behavior indicates that $D_{s t}$ is a multifractional process. A method to describe the multiple scaling of the measure representation of the $D_{s t}$ time series was provided in Wanliss et al. (2005). A prediction method was detailed in Anh et al. (2005) together with some evaluation of its performance. A two-dimensional chaos game representation of the $D_{s t}$ index for prediction of geomagnetic storm events was proposed in Yu et al. (2007). The spatiotemporal scaling properties of the ground geomagnetic field variations from individual magnetometer stations were studied in Pulkkinen et al. (2005) and Cersosimo and Wanliss (2007).

The detrended fluctuation analysis (DFA) introduced by Peng et al. (1994) has become a widely used technique to determine the fractal scaling properties of, and to detect the long-range correlations in, stationary and nonstationary time series (Hu et al., 2001; Chen et al., 2002). For uncorrelated data, the scaling exponent $h$ in DFA is equal to $1 / 2$. The range $1 / 2<h<1.0$ indicates the presence of long memory or persistence, while the range $0<h<1 / 2$ indicates short memory or anti-persistence. The DFA method has been applied successfully in diverse fields such as DNA sequences, heart rate dynamics, neuron spiking, weather records, economics time series (Kantelhardt et al., 2002, and the references therein). Yu et al. (2001) used it to study the length

Published by Copernicus Publications on behalf of the European Geosciences Union and the American Geophysical Union. 
sequences of complete genomes. Recently, Yu et al. (2006) used the DFA method to study the classification problem of protein secondary structures. The multifractal detrended fluctuation analysis (MF-DFA) proposed by Kantelhardt et al. (2002) is a modified version of DFA to detect multifractal properties of time series. It allows a reliable multifractal characteriation of nonstationary time series typical of geophysical phenomena (Kantelhardt et al., 2002). Movahed et al. (2006) used the MF-DFA to study sunspot fluctuations. In this paper, we analyze ground magnetic fluctuations for the year 2000 Anno Domini through the use of MF-DFA.

\section{Multifractal detrended fluctuation analysis}

Simple multifractal analyses have been developed for the multifractal characterization of normalized, stationary time series. This standard formalism does not give correct results for nonstationary time series which are affected by trends or cannot be normalized. Multifractal detrended fluctuation analysis, which is a generalization of the standard DFA, is based on the identification of the scaling of the $q$ th-order moments of the time series, which may be nonstationary.

We first recapture some features of this technique. Consider a time series $\left\{X_{1}, X_{2}, \ldots, X_{N}\right\}$ of length $N$. For an integer $s \geq 0$, we divide the time series into $[N / s]$ segments of equal length $s$, where $[N / s]$ is the integer part of $N / s$. In each segment $j$, we compute the partial sums $Y(i)=\sum_{k=1}^{i} X_{k}, i=1,2, \ldots, s$, fit a local trend $y_{j}(i)$ to $Y(i)$ by least squares, then compute the sample variances of the residuals:

$$
\begin{aligned}
F^{2}(s, j)=\frac{1}{s} \sum_{i=1}^{s}\left(Y((j-1) s+i)-y_{j}(i)\right)^{2}, & \\
& j=1, \ldots,[N / s] .
\end{aligned}
$$

The $q$ th-order fluctuation function is then defined as the average over all segments:

$F_{q}(s)=\left(\frac{1}{[N / s]} \sum_{j=1}^{[N / s]}\left(F^{2}(s, j)\right)^{q / 2}\right)^{1 / q}$.

Since the segments are all of the same length, the secondorder fluctuation function $F_{2}(s)$ is equivalent to the sample variance of the entire series. This is not so for the general case $q \neq 2$. We will assume that $F_{q}(s)$ is characterized by a power law:

$F_{q}(s) \propto s^{h(q)}$.

The scaling function $h(q)$ is then determined by the regression of $\log F_{q}(s)$ on $\log s$ in some range of time scale $s$.

Fractional Brownian motion $\left\{B_{H}(t), t \geq 0\right\}$ is a Gaussian process with stationary increments, having mean 0 , variance $E\left(B_{H}^{2}(t)\right)=t^{2 H}$ and covariance

$$
E\left(B_{H}(s) B_{H}(t)\right)=\frac{1}{2}\left(s^{2 H}+t^{2 H}-|s-t|^{2 H}\right) .
$$

It is statistically self-similar in the sense that $\left\{B_{H}(a t), t \geq 0\right\}$ has the same finite-dimensional distributions as $\left\{a^{H} B_{H}(t), t \geq 0\right\}$ for all $a>0$. The self-similarity parameter $H$, also known as the Hurst index, takes values between 0 and $1 . B_{H}(t)$ possesses long-range dependence or long memory when $1 / 2<H<1$. The spectral density of its increments is

$f(\omega)=C_{H}|\omega|^{1-2 H}$ as $|\omega| \rightarrow 0$,

where $C_{H}$ is a constant (Flandrin, 1989; Taqqu et al., 1995).

For the increments of fractional Brownian motion, and using a linear trend $y_{j}(i)=a_{j}+b_{j} i$ in Eq. (1), Taqqu et al. (1995) showed that when $s \rightarrow \infty$, the expectation of their sample variance $F_{2}(s)$ as given by Eq. (2) with $q=2$ is asymptotically proportional to $s^{2 H}$ :

$E\left(F^{2}(s)\right) \sim\left(\frac{2}{2 H+1}+\frac{1}{H+2}-\frac{2}{H+1}\right) s^{2 H}$.

This result suggests that the Hurst index $H$ may be obtained from the slope of the log-regression based on Eq. (3) for $q=2$, that is, $H=h$ (2) for the increments of fractional Brownian motion.

For fractional Brownian motion itself and also using a linear trend in Eq. (1), Movahed et al. (2006) showed that

$E\left(F^{2}(s)\right) \sim C_{H} s^{2(H+1)}$ as $s \rightarrow \infty$,

where

$$
\begin{aligned}
C_{H}= & \frac{1}{(2 H+3)(H+1)^{2}}-\frac{4}{(H+1)^{2}(H+2)^{2}} \\
& -\frac{12}{(H+1)^{2}(H+3)^{2}}+\frac{12}{(H+1)^{2}(H+2)(H+3)} .
\end{aligned}
$$

Thus, for fractional Brownian motion, we infer $H=h(2)-1$. Using this relationship (or $H=h$ (2) for the stationarity case) and the estimate of $h(2)$ from the regression of $\log F_{2}(s)$ on $\log s$, an estimate of the Hurst index $H$, and hence the extent of long memory in the time series, is obtained.

Note that linear, quadratic, cubic or higher order polynomials can be used in the local trend fitting, and the DFA is accordingly called DFA1, DFA2, DFA $3, \ldots$ In the following we use only DFA 1 .

We generated a time series of Gaussian noise and a sample path of Brownian motion with the same length as our data. Gaussian noise is uncorrelated, while Brownian motion has uncorrelated increments, hence both have Hurst index $H=1 / 2$. We then computed the exponents $h(1)$ and $h(2)$ using the MF-DFA1. The fitting of the exponents is shown in Fig. 1. The estimated values of $h(2)$ are $0.4960 \pm 0.0049$ for the Gaussian noise series and 1.4979 \pm 0.0046 for the Brownian motion series for both small and large time scales. These numerical results support the above relationships between the exponents $H$ and $h(2)$.

For a stationary, normalized series, the exponent $h(q)$ for small scales is directly related to the scaling exponent $\tau(q)$ 

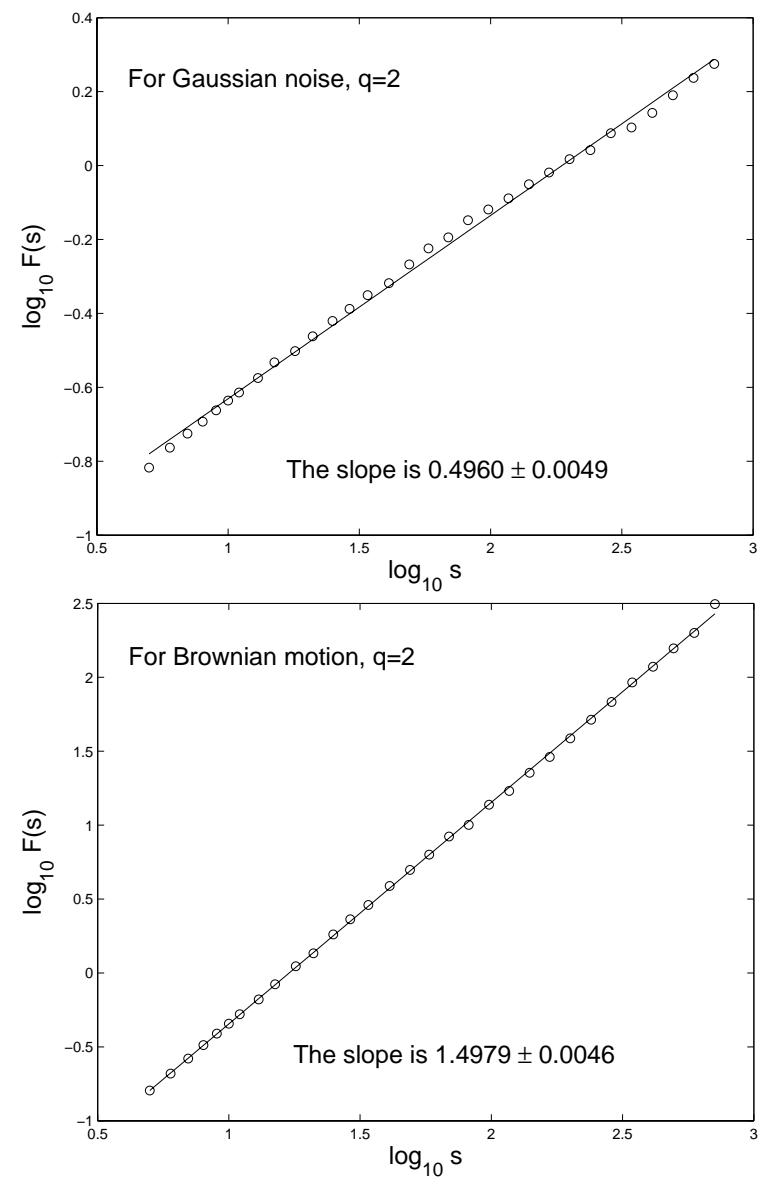

Fig. 1. Log-log plots of $F_{q}(s)$ versus $s$ for the Gaussian noise time series (top) and Brownian motion (bottom) with $q=2$.

defined by the standard partition function-based multifractal formalism. The relation between this exponent $h(q)$ and the generalized multifractal dimension is given by (Movahed et al., 2006)

$D(q)=\frac{\tau(q)}{q-1}=\frac{q h(q)-1}{q-1}$.

But the standard multifractal formalism does not give correct results for nonstationary time series that are affected by trends or cannot be normalized (Movahed et al., 2006). The MF-DFA seems to be a suitable for this situation (Kantelhardt et al., 2002). Hence in this work, we use the $h(q)$ curves to characterize the multifractal property of magnetic field time series.

\section{Results and discussion}

We use the MF-DFA method to analyze the hourly averaged magnetic field time series $B_{x}, B_{y}, B_{z}$ from INTERMAGNET stations. We break the stations down into 6 groups for the year 2000 A.D.: Southwest North America (NA1), North-
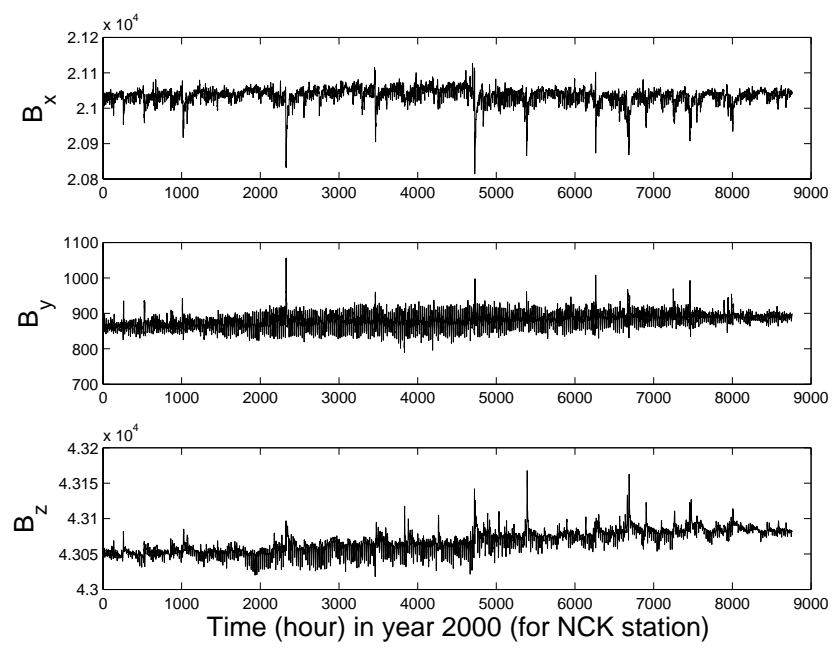

Fig. 2. The hourly magnetic field data for the year 2000 at station NCK.

Table 1. The estimated values of $a_{0}, a_{1}$ and $a_{2}$ corresponding to $q=2$ for the $B_{x}$ component at the selected stations.

\begin{tabular}{ccccc}
\hline Group & Station & $a_{0}$ & $a_{1}$ & $a_{2}$ \\
\hline \multirow{4}{*}{ NA1 } & BOU & $1.1944 \pm 0.0327$ & $0.9011 \pm 0.0209$ & $0.8911 \pm 0.0244$ \\
& FRN & $1.2199 \pm 0.0265$ & $1.0203 \pm 0.0217$ & $0.8832 \pm 0.0237$ \\
& DLR & $1.2070 \pm 0.0206$ & $1.0639 \pm 0.0292$ & $0.8930 \pm 0.0248$ \\
& TUC & $1.2478 \pm 0.0241$ & $1.0697 \pm 0.0269$ & $0.8845 \pm 0.0251$ \\
\hline \multirow{4}{*}{ NA2 } & FCC & $1.2150 \pm 0.0225$ & $0.3614 \pm 0.0130$ & $0.5621 \pm 0.0088$ \\
& PBQ & $1.2249 \pm 0.0218$ & $0.3758 \pm 0.0101$ & $0.5922 \pm 0.0110$ \\
& OTT & $1.1733 \pm 0.0722$ & $0.5659 \pm 0.0291$ & $0.7645 \pm 0.0187$ \\
& STJ & $1.2188 \pm 0.0531$ & $0.5708 \pm 0.0434$ & $0.8841 \pm 0.0212$ \\
\hline \multirow{5}{*}{ CEUR } & BDV & $1.3191 \pm 0.0300$ & $0.9445 \pm 0.0325$ & $0.8816 \pm 0.0220$ \\
& NCK & $1.3010 \pm 0.0291$ & $0.9916 \pm 0.0299$ & $0.8852 \pm 0.0226$ \\
& FUR & $1.3148 \pm 0.0275$ & $0.9660 \pm 0.0326$ & $0.8843 \pm 0.0223$ \\
& NGK & $1.2998 \pm 0.0362$ & $0.8360 \pm 0.0343$ & $0.8726 \pm 0.0208$ \\
\hline \multirow{5}{*}{ NEUR } & ABK & $1.2200 \pm 0.0292$ & $0.3520 \pm 0.0141$ & $0.4878 \pm 0.0227$ \\
& NUR & $1.2058 \pm 0.0672$ & $0.6042 \pm 0.0559$ & $0.7177 \pm 0.0197$ \\
& LOV & $1.2167 \pm 0.0664$ & $0.6329 \pm 0.0535$ & $0.7489 \pm 0.0204$ \\
& SOD & $1.2122 \pm 0.0319$ & $0.3965 \pm 0.0164$ & $0.5122 \pm 0.0234$ \\
\hline \multirow{5}{*}{ ASIA } & ASP & $1.3426 \pm 0.0171$ & $1.1102 \pm 0.0152$ & $0.9065 \pm 0.0205$ \\
& CTA & $1.3430 \pm 0.0227$ & $1.1205 \pm 0.0189$ & $0.8772 \pm 0.0233$ \\
& KDU & $1.4687 \pm 0.0468$ & $0.9011 \pm 0.0383$ & $0.8356 \pm 0.0295$ \\
\hline & MMK & $1.2653 \pm 0.0330$ & $1.0600 \pm 0.0263$ & $0.8958 \pm 0.0237$ \\
& & $1.3146 \pm 0.0273$ & $1.1553 \pm 0.0287$ & $0.9019 \pm 0.0254$ \\
& & $1.2761 \pm 0.0367$ & $1.0058 \pm 0.0231$ & $0.9030 \pm 0.0238$ \\
\hline
\end{tabular}

east North America (NA2), Central Europe (CEUR), Northern Europe (NEUR), Australasia (AUS) and Asia (ASIA). The stations in each group are listed in Table 1. Figure 2 shows the hourly magnetic field data from the NCK station in CEUR, and illustrates the anomalous nature of this kind of data. The time series appear to be non-Gaussian and display Brownian motion-type behavior at small scales. This indicates the existence of different scalings in the process.

Following the MF-DFA1 method, generalized exponents $h(q)$ can be found by analyzing log-log plots of 

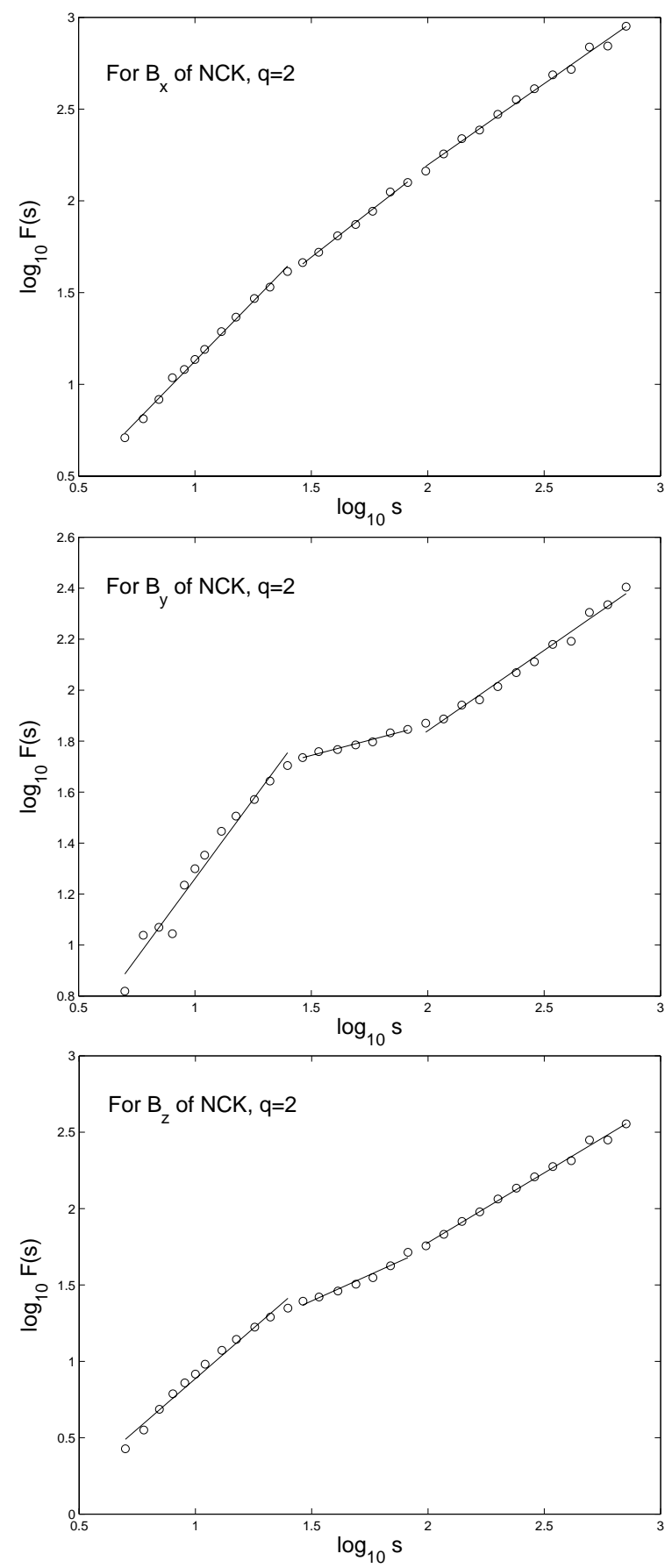

Fig. 3. Crossover behavior of $\log -\log$ plots of $F_{q}(s)$ versus $s$ for the hourly magnetic field components $B_{x}, B_{y}, B_{z}$ at station NCK.

$F_{q}(s)$ mboxversus $s$ for each $q$. Our investigation shows that there are three scaling regimes in the $q$ th-order fluctuation function. The log-log plots of fluctuation functions with $q=2$ for the hourly averaged magnetic field data of station NCK are shown in Fig. 3. The $q$ th-order fluctuation functions have a clear point at which the first scaling region switches to a new region with a new scaling exponent. We call this point
Table 2. The estimated values of $a_{0}, a_{1}$ and $a_{2}$ corresponding to $q=2$ for the $B_{y}$ component at the selected stations.

\begin{tabular}{ccccc}
\hline Group & Station & $a_{0}$ & $a_{1}$ & $a_{2}$ \\
\hline \multirow{3}{*}{ NA1 } & BOU & $1.2027 \pm 0.0636$ & $0.1838 \pm 0.0096$ & $0.6898 \pm 0.0344$ \\
& FRN & $1.2831 \pm 0.0823$ & $0.2465 \pm 0.0187$ & $0.7790 \pm 0.0187$ \\
& DLR & $1.1932 \pm 0.0672$ & $0.1682 \pm 0.0103$ & $0.6362 \pm 0.0345$ \\
& TUC & $1.2268 \pm 0.0720$ & $0.2177 \pm 0.0155$ & $0.7618 \pm 0.0236$ \\
\hline \multirow{4}{*}{ NA2 } & FCC & $1.0880 \pm 0.0390$ & $0.2811 \pm 0.0228$ & $0.4254 \pm 0.0087$ \\
& PBQ & $1.2273 \pm 0.0321$ & $0.2918 \pm 0.0170$ & $0.4752 \pm 0.0170$ \\
& OTT & $1.1402 \pm 0.0454$ & $0.2489 \pm 0.0200$ & $0.4266 \pm 0.0182$ \\
& STJ & $1.2398 \pm 0.0466$ & $0.2268 \pm 0.0239$ & $0.4887 \pm 0.0353$ \\
\hline \multirow{4}{*}{ CEUR } & BDV & $1.2488 \pm 0.0662$ & $0.2862 \pm 0.0225$ & $0.6782 \pm 0.0219$ \\
& NCK & $1.2423 \pm 0.0706$ & $0.2400 \pm 0.0178$ & $0.6318 \pm 0.0245$ \\
& FUR & $1.2457 \pm 0.0637$ & $0.2829 \pm 0.0218$ & $0.6361 \pm 0.0201$ \\
& NGK & $1.2505 \pm 0.0629$ & $0.2871 \pm 0.0230$ & $0.6747 \pm 0.0222$ \\
\hline \multirow{5}{*}{ NEUR } & ABK & $1.3101 \pm 0.0259$ & $0.2570 \pm 0.0118$ & $0.4935 \pm 0.0124$ \\
& NUR & $1.2780 \pm 0.0542$ & $0.2947 \pm 0.0268$ & $0.6455 \pm 0.0177$ \\
& LOV & $1.2799 \pm 0.0541$ & $0.2983 \pm 0.0250$ & $0.6644 \pm 0.0201$ \\
& SOD & $1.3066 \pm 0.0263$ & $0.2819 \pm 0.0153$ & $0.5439 \pm 0.0176$ \\
\hline \multirow{5}{*}{ AUS } & ASP & $1.1481 \pm 0.0602$ & $0.1798 \pm 0.0118$ & $0.4536 \pm 0.0313$ \\
& CTA & $1.1843 \pm 0.0649$ & $0.2026 \pm 0.0132$ & $0.5450 \pm 0.0305$ \\
& KDU & $1.1284 \pm 0.0630$ & $0.2162 \pm 0.0114$ & $0.5064 \pm 0.0228$ \\
\hline & BMT & $1.1458 \pm 0.0613$ & $0.1206 \pm 0.0099$ & $0.4424 \pm 0.0354$ \\
& KAK & $1.2649 \pm 0.0803$ & $0.1572 \pm 0.0110$ & $0.4823 \pm 0.0278$ \\
& MMB & $1.3302 \pm 0.0843$ & $0.1530 \pm 0.0122$ & $0.4801 \pm 0.0283$ \\
\hline
\end{tabular}

a break point. We find the break point occurs around a time scale of approximately $23 \mathrm{~h}$ and it is universal for all components of the selected stations. The ubiquity of this break point can be associated with the regular diurnal quiet solar variation in the current system.

In order to determine statistical properties of the fluctuations, and to see the extent to which the fluctuations are reflected in terms of geographical location, we compute the three scaling exponents in small time scales, middle time scales and large time scales of second-order fluctuation functions for the selected data sets. For each $q$, we denote the exponent $h(q)$ in small time scales as $a_{0}$, that in middle time scales as $a_{1}$ and that in large time scales as $a_{2}$. We list the estimated values of $a_{0}, a_{1}$ and $a_{2}$ for $q=2$ of the $B_{x}$ component of these stations in Table 1 , the values for $q=2$ of the $B_{y}$ component in Table 2, and the values for $q=2$ of the $B_{z}$ component in Table 3. The values in the first columns of Tables 1 to 3 confirm that the magnetic field components $B_{x}$, $B_{y}$ and $B_{z}$ are nonstationary processes with anti-persistent correlations.

We find that the parameters $a_{0}$ and $a_{2}$ corresponding to $q=2$ for field components $B_{x}, B_{y}, B_{z}$ reflect the regional character of the stations, suggesting that these scaling exponents can be used for station classification. In Fig. 4, we show the three-dimensional parameter space spanned by $a_{2}(q=2)$ for $B_{x}, a_{2}(q=2)$ for $B_{y}$ and $a_{2}(q=2)$ for $B_{z}$. A point in this space represents a magnetometer station. According to this figure, the stations can be divided into four groups: NA2 stations, NEUR stations, NA1 and CEUR stations, ASIA and AUS stations. In order to clearly distinguish 
Table 3. The estimated values of $a_{0}, a_{1}$ and $a_{2}$ corresponding to $q=2$ for the $B_{z}$ component at the selected stations.

\begin{tabular}{|c|c|c|c|c|}
\hline Group & Station & $a_{0}$ & $a_{1}$ & $a_{2}$ \\
\hline \multirow{4}{*}{ NA1 } & BOU & $1.2247 \pm 0.0461$ & $0.7091 \pm 0.0384$ & $0.8619 \pm 0.0192$ \\
\hline & FRN & $1.2389 \pm 0.0611$ & $0.5142 \pm 0.0167$ & $1.0867 \pm 0.0367$ \\
\hline & DLR & $1.2544 \pm 0.0487$ & $0.3972 \pm 0.0323$ & $1.0248 \pm 0.0270$ \\
\hline & TUC & $1.2699 \pm 0.0597$ & $0.5230 \pm 0.0258$ & $0.8981 \pm 0.0158$ \\
\hline \multirow{4}{*}{ NA2 } & FCC & $1.1543 \pm 0.0355$ & $0.4637 \pm 0.0082$ & $0.6703 \pm 0.0165$ \\
\hline & PBQ & $1.0146 \pm 0.0320$ & $0.5446 \pm 0.0242$ & $0.7510 \pm 0.0173$ \\
\hline & OTT & $1.1106 \pm 0.0283$ & $0.4389 \pm 0.0210$ & $0.6979 \pm 0.0270$ \\
\hline & STJ & $1.2967 \pm 0.0388$ & $0.3296 \pm 0.0375$ & $0.7518 \pm 0.0204$ \\
\hline \multirow{4}{*}{ CEUR } & $\mathrm{BDV}$ & $1.3104 \pm 0.0562$ & $0.6672 \pm 0.0622$ & $0.9257 \pm 0.0204$ \\
\hline & NCK & $1.3213 \pm 0.0558$ & $0.6860 \pm 0.0630$ & $0.9134 \pm 0.0212$ \\
\hline & FUR & $1.3118 \pm 0.0524$ & $0.6665 \pm 0.0615$ & $0.9236 \pm 0.0223$ \\
\hline & NGK & $1.2756 \pm 0.0615$ & $0.5785 \pm 0.0559$ & $0.9016 \pm 0.0200$ \\
\hline \multirow{4}{*}{ NEUR } & $\mathrm{ABK}$ & $1.0453 \pm 0.0389$ & $0.5048 \pm 0.0334$ & $0.6280 \pm 0.0137$ \\
\hline & NUR & $1.2550 \pm 0.0356$ & $0.4563 \pm 0.0444$ & $0.5918 \pm 0.0218$ \\
\hline & LOV & $1.2834 \pm 0.0436$ & $0.4461 \pm 0.0506$ & $0.6363 \pm 0.0234$ \\
\hline & SOD & $1.0931 \pm 0.0343$ & $0.4419 \pm 0.0214$ & $0.6117 \pm 0.0120$ \\
\hline \multirow{3}{*}{ AUS } & ASP & $1.4287 \pm 0.0854$ & $0.4486 \pm 0.0360$ & $0.8817 \pm 0.0205$ \\
\hline & CTA & $1.3476 \pm 0.0506$ & $0.4400 \pm 0.0272$ & $0.8413 \pm 0.0223$ \\
\hline & $\mathrm{KDU}$ & $1.4610 \pm 0.0899$ & $0.3460 \pm 0.0308$ & $0.9153 \pm 0.0250$ \\
\hline \multirow{3}{*}{ ASIA } & BMT & $1.4274 \pm 0.0915$ & $0.3756 \pm 0.0367$ & $0.8588 \pm 0.0204$ \\
\hline & KAK & $1.2057 \pm 0.0486$ & $0.3127 \pm 0.0350$ & $0.8135 \pm 0.0201$ \\
\hline & MMB & $1.2926 \pm 0.0488$ & $0.5152 \pm 0.0458$ & $0.9710 \pm 0.0262$ \\
\hline
\end{tabular}

NA1 stations from CEUR stations, and ASIA stations from AUS stations, we find that two-dimensional parameter spaces spanned by $a_{0}(q=2)$ for $B_{x}$ and $a_{0}(q=2)$ for $B_{y}$ can be used. The results are shown in Fig. 5.

In order to give a quantitative assessment of our clustering on the selected stations, we use Fisher's linear discriminant algorithm (Mardia et al., 1979; Duda et al., 2001) to calculate the discriminant accuracies of the following procedure:

(i) Separating NA2 and NEUR stations from CEUR, NA1, AUS and ASIA stations in Fig. 4;

(ii) Separating NA2 stations from NEUR stations in Fig. 4;

(iii) Separating NA1 and CEUR stations from AUS and ASIA stations in Fig. 4;

(iv) Separating NA1 stations from CEUR stations in Fig. 5;

(v) Separating AUS stations from ASIA stations in Fig. 5.

Fisher's discriminant algorithm is used to find a classifier in the two- or three-dimensional space for the training set. The given training set $H=\left\{\mathbf{x}_{1}, \mathbf{x}_{2}, \ldots, \mathbf{x}_{n}\right\}$ is partitioned into $n_{1} \leq n$ training vectors in a subset $H_{1}$ and $n_{2} \leq n$ training vectors in a subset $H_{2}$, where $n_{1}+n_{2}=n$ and each vector $\boldsymbol{x}_{i}$ is a point in the two- or three-dimensional parameter space. Then $H=H_{1} \cup H_{2}$. We need to find a parameter vector $\boldsymbol{w}=\left(w_{1}, w_{2}, w_{3}\right)$ (for three-dimensional space) and $\boldsymbol{w}=\left(w_{1}, w_{2}\right)$ (for two-dimensional space) such that

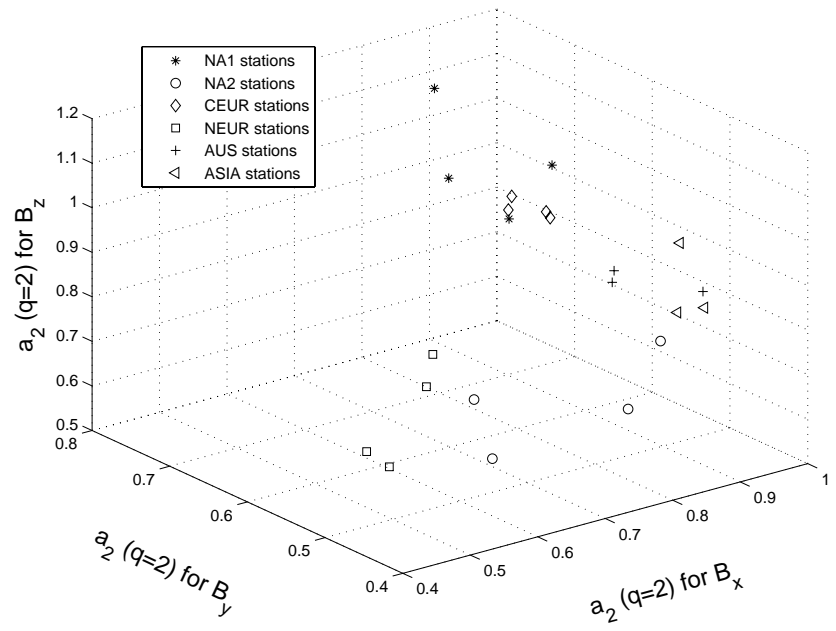

Fig. 4. Classification of stations in the three-dimensional parameter space spanned by $a_{2}(q=2)$ for $B_{x}, a_{2}(q=2)$ for $B_{y}$ and $a_{2}(q=2)$ for $B_{z}$.
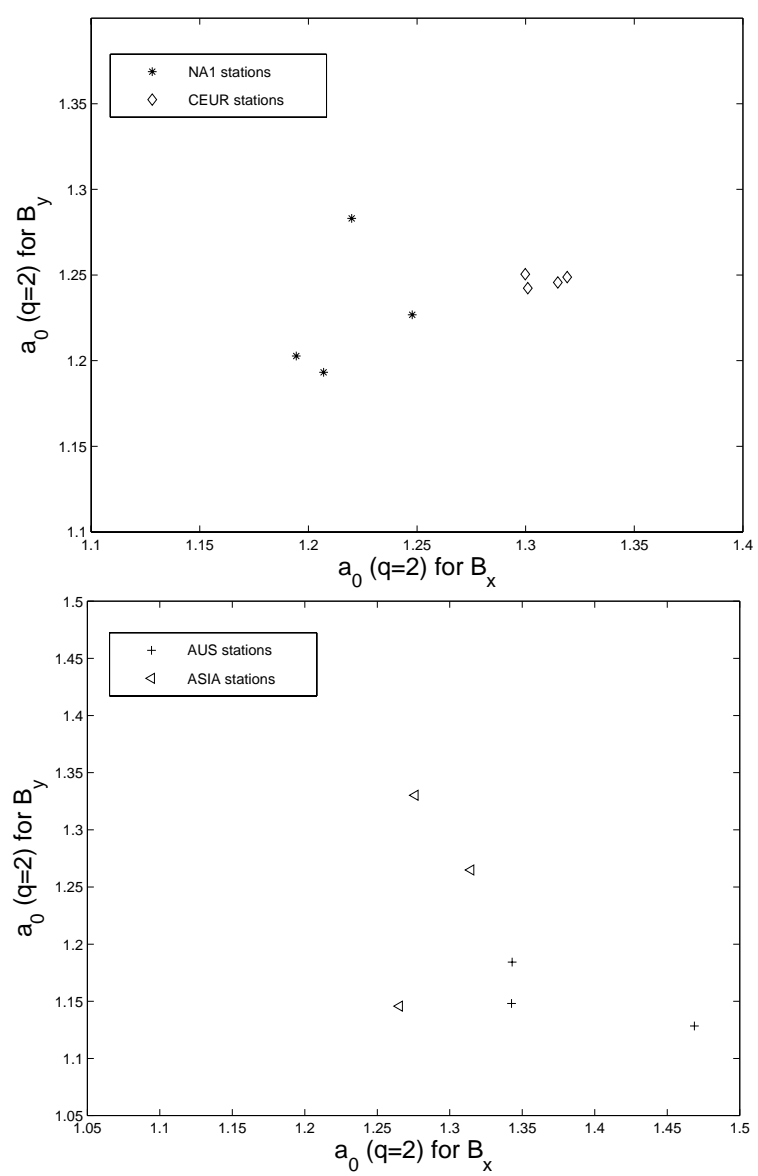

Fig. 5. Classification of stations in the two-dimensional parameter space spanned by $a_{0}(q=2)$ for $B_{x}$ and $a_{0}(q=2)$ for $B_{y}$. 
Table 4. The parameters in Fisher's discriminant algorithm and the discriminant accuracies for stations in the present method.

\begin{tabular}{lrrrcc}
\hline Stations & $w_{1}$ & $w_{2}$ & $w_{3}$ & $p_{H 1}$ & $p_{H 2}$ \\
\hline step (i) & -1.2197 & -0.0142 & -2.3253 & $87.5 \%$ & $100 \%$ \\
step (ii) & 4.5762 & -15.8702 & 19.1322 & $100 \%$ & $100 \%$ \\
step (iii) & 5.4250 & 7.3880 & 0.1395 & $100 \%$ & $100 \%$ \\
step (iv) & -53.6141 & 7.4140 & & $100 \%$ & $100 \%$ \\
step (v) & 7.8468 & -4.3222 & & $100 \%$ & $100 \%$ \\
\hline
\end{tabular}

$\left\{y_{i}=\boldsymbol{w}^{T} \boldsymbol{x}_{i}\right\}_{i=1}^{n}$ can be classified into two classes in the space of real numbers. If we denote

$\mathbf{m}_{j}=\frac{1}{n_{j}} \sum_{\boldsymbol{x}_{i} \in H_{j}} \boldsymbol{x}_{i}, \quad j=1,2$,

$\mathbf{S}_{j}=\sum_{\boldsymbol{x}_{i} \in H_{j}}\left(\boldsymbol{x}_{i}-\mathbf{m}_{j}\right)\left(\boldsymbol{x}_{i}-\mathbf{m}_{j}\right)^{T}, \quad j=1,2$,

$\mathbf{S}_{w}=\mathbf{S}_{1}+\mathbf{S}_{2}$

then the parameter vector $\boldsymbol{w}$ is estimated as $\mathbf{S}_{w}^{-1}\left(\mathbf{m}_{1}-\mathbf{m}_{2}\right)$ (Duda et al., 2001). As a result, Fisher's discriminant rule becomes

$$
\begin{aligned}
& \text { "assign } \mathbf{x} \text { to } H_{1} \text { if }\left(\mathbf{m}_{1}-\mathbf{m}_{2}\right)^{T} \mathbf{C}_{w}^{-1}\left[\mathbf{x}-\frac{1}{2}\left(\mathbf{m}_{1}+\right.\right. \\
& \left.\left.\mathbf{m}_{2}\right)\right]>0 \text { and to } H_{2} \text { otherwise." (Mardia et al., } \\
& \text { 1979). }
\end{aligned}
$$

We use the whole data set as the training set here because the number of stations is small. The discriminant accuracies for resubstitution analysis are defined as

$$
\begin{aligned}
& p_{H 1}=\operatorname{num}_{c h 1} / n_{1}, \\
& p_{H 2}=\operatorname{num}_{c h 2} / n_{2},
\end{aligned}
$$

where num ${ }_{c h 1}$ and num ${ }_{c h 2}$ mean the number of correctly discriminated $H_{1}$ elements and the number of correctly discriminated $\mathrm{H}_{2}$ elements in the training set respectively.

We denote the NA2 and NEUR stations as $H_{1}$, the stations in the other groups as $H_{2}$ in steps (i); the NA2 stations as $H_{1}$, the NEUR stations as $H_{2}$ in steps (ii); the NA1 and CEUR stations as $H_{1}$, the AUS and ASIA stations as $H_{2}$ in steps (iii); the NA1 stations as $H_{1}$, the CEUR stations as $H_{2}$ in step (iv); the AUS stations as $H_{1}$, the ASIA stations as $H_{2}$ in step (v).

The estimated parameters $\boldsymbol{w}=\left(w_{1}, w_{2}, w_{3}\right)$ or $\boldsymbol{w}=\left(w_{1}, w_{2}\right)$ in Fisher's discriminant algorithm and the discriminant accuracies for the stations in the above five steps are given in Table 4. It is seen that the discriminant accuracies are very high.

In order to find whether the magnetic field time series have multifractal nature, we calculate the exponents $h(q)$ of small scales for all stations. The nonlinearity of the $h(q)$ curves obtained suggests that all the magnetic field time series have multifractal property. For example, we shown those of the stations from the NA1 and AUS groups in Figs. 6 and 7.
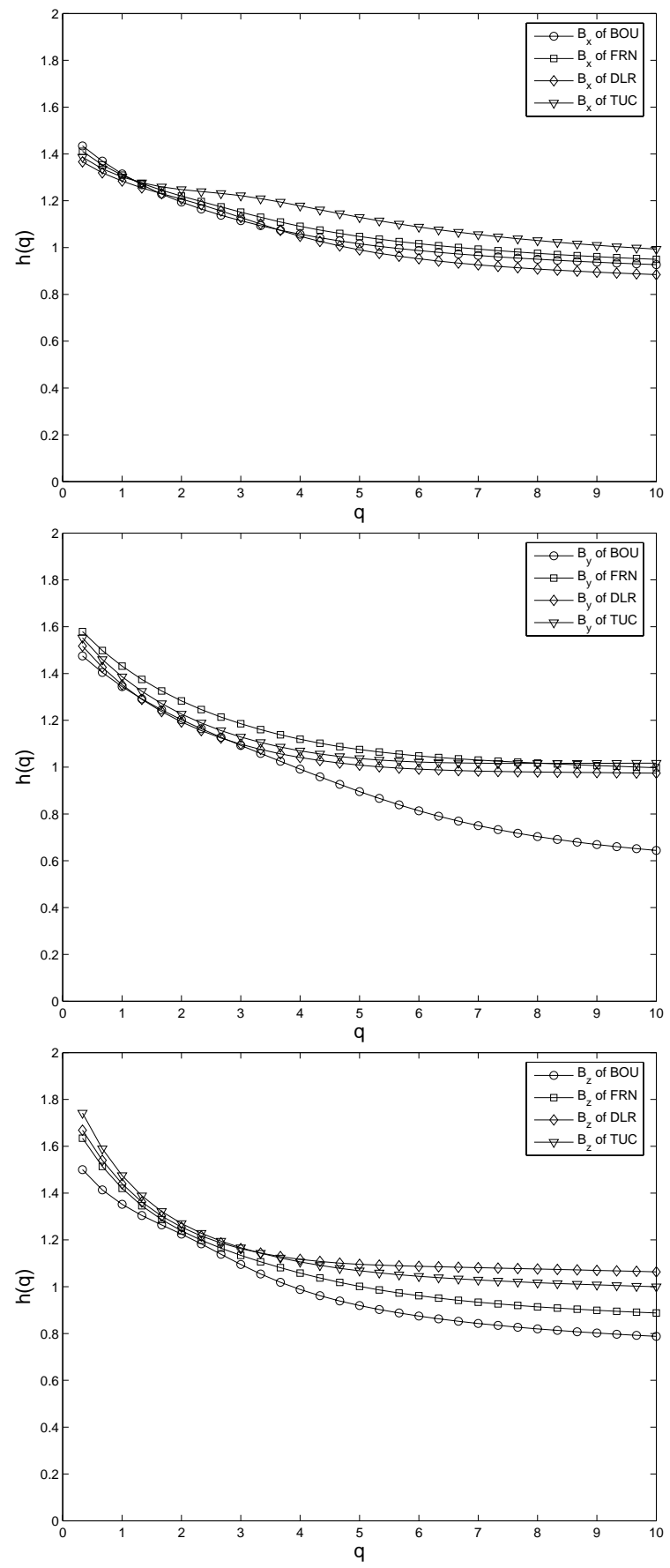

Fig. 6. The exponents $h(q)$ of small time scales for the NA1 stations.

\section{Conclusions}

Many time series do not exhibit simple monofractal scaling behavior, which can be characterized by a single exponent. In some cases, there exist crossover (time-) scales separating regimes with different scaling exponents. Hence a multitude of scaling exponents is required for a full description 

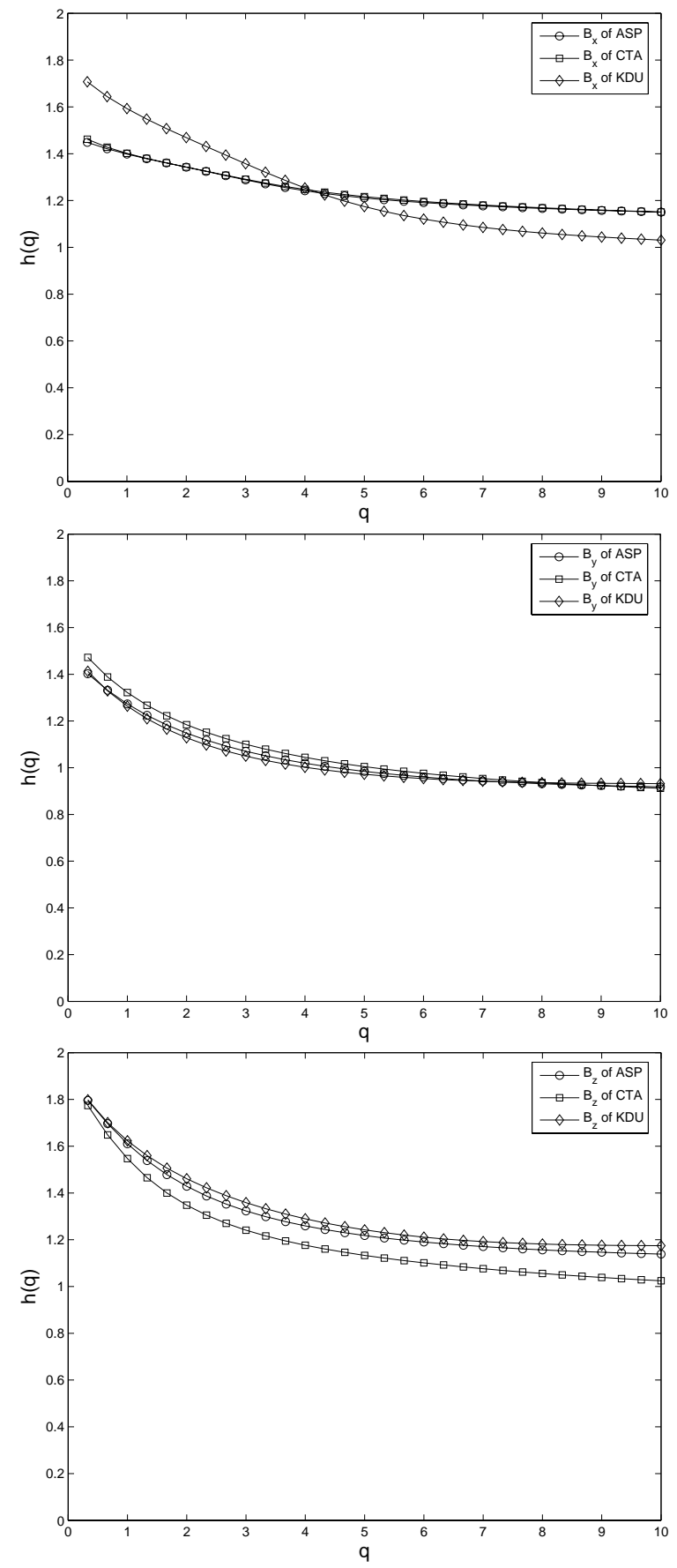

Fig. 7. The exponents $h(q)$ of small time scales for the AUS stations.

of the scaling behavior, and a multifractal analysis must be applied (Kantelhardt et al., 2002). Simple multifractal analyses have been developed for the multifractal characterization of normalized, stationary time series. This standard formalism does not give correct results for nonstationary time series which are affected by trends or cannot be normalized. The MF-DFA method is a modified version of the DFA adapted to nonstationary processes to detect their multifractal properties.

From the MF-DFA estimation, we find that there are three scaling regimes in the $q$ th-order fluctuation functions of all hourly averaged magnetic field time series. The $q$ th-order fluctuation functions have a clear point at which the first crossover time scales change to the second crossover time scales. For $q=1,2$, the break point occurs around the time scale of $23 \mathrm{~h}$ and is universal for all stations. The break point can be associated with the regular diurnal quiet solar variation of the current system.

From Tables 1 to 3 , the $h(2)$ values of small time scales confirm that magnetic field components $B_{x}, B_{y}$ and $B_{z}$ are nonstationary processes with anti-persistent correlations. The $h(q)$ curves obtained indicate that all the magnetic field time series have multifractal property.

The result that is most useful for spatiotemporal space weather modelling is that the parameters $a_{0}$ and $a_{2}$ corresponding to $q=2$ for field components $B_{x}, B_{y}$ and $B_{z}$ reflect the regional character of the global INTERMAGNET stations. Therefore these exponents can be used for station classification. The discriminant accuracies are very high indicating the robustness of this result. The short period and long period scaling regimes exhibit similar fractal scaling within limited geographical regions (e.g. Figs. 3 and 5). This suggests that a feasible strategy to develop spatiotemporal space weather models is to first develop suitable models for homogeneous subregions, then couple them into a global multiscale model.

Acknowledgements. This research was partially supported by the Australian Research Council grant DP0559807, Natural Science Foundation of China grant 30570426, Fok Ying Tung Education Foundation grant 101004, the Youth Foundation of Educational Department of Hunan Province grant 05B007. The material is based on work supported by the National Science Foundation under grants ATM-0449403, DMS-0417690 and DMS-0417676.

Edited by: P. Yiou

Reviewed by: two anonymous referees

\section{References}

Anh, V. V., Yu, Z. G., Wanliss, J. A., and Watson, S. M.: Prediction of magnetic storm events using the $D_{s t}$ index, Nonlin. Processes Geophys., 12, 799-806, 2005, http://www.nonlin-processes-geophys.net/12/799/2005/.

Beran, J.: Statistics for long-memory processes, Chapman \& Hall, NY, 1994.

Burlaga, L. F.: Multifractal structure of the interplanetary magnetic field: Voyager 2 observations near 25 AU, 1987-1988, Geophys. Res. Lett., 18(1), 69-72, 1991.

Burlaga, L. F.: Lognormal and multifractal distributions of the heliospheric magnetic field, J. Geophys. Res., 106, 15 917-15927, 2001. 
Burlaga, L. F., Wang, C., and Ness, N. F.: A model and observations of the multifractal spectrum of the heliospheric magnetic field strength fluctuations near $40 \mathrm{AU}$, Geophys. Res. Lett., 30, doi:10.1029/2003GL016903, 2003.

Chen, Z., Ivanov, P. Ch., Hu, K., and Stanley, H. E.: Effect of nonstationarities on detrended fluctuation analysis, Phys. Rev. E, 65, 041107, 2002.

Cersosimo, D. O. and Wanliss, J. A.: Initial studies of high latitude magnetic field data during different magnetospheric conditions, Earth Planets Space, 59(1), 39-43, 2007.

Duda, R. O., Hart, P. E., and Stork, D. G.: Pattern Classification, Second Edition, New York, John Wiley \& Sons, Inc, 2001.

Flandrin, P.: On the spectrum of fractional Brownian motions, IEEE Trans. Info. Theory, 35, 197-199, 1989.

Hu, K., Ivanov, P. Ch., Chen, Z., Carpena, P., and Stanley, H. E.: Effect of trends on detrended fluctuation analysis, Phys. Rev. E, 64, 011114, 2001.

Kabin, K. and Papitashvili, V. O.: Fractal properties of the IMF and the Earth's magnetotail field, Earth Planets Space, 50, 87-90, 1998.

Kantelhardt, J. W., Zschiegner, S. A., Koscielny-Bunde, E., Bunde, A., Havlin, S., and Stanley, H. E.: Multifractal detrended fluctuation analysis of nonstationary time series, Physica A, 316, 87114, 2002.

Lui, A. T. Y., Chapman, S. C., Liou, K., Newell, P. T., Meng, C. I., Brittnacher, M., and Parks, G. K.: Is the dynamic magnetosphere an avalanching system?, Geophys. Res. Lett., 27, 911-914, 2000.

Lui, A. T. Y.: Multiscale phenomena in the near-Earth magnetosphere, J. Atmos. Sol.-Terr. Phys., 64, 125-143, 2002.

Lui, A. T. Y., Lai, W. W., Liou, K., and Meng, C. I.: A new technique for short-term forecast of auroral activity, Geophys. Res. Lett., 30, art. no. 1258, 2003.

Mardia, K. V., Kent, J. T., and Bibby, J. M.: Multivariate Analysis, London, Academic Press, 1979.
Movahed, M. S., Jafari, G. R., Ghasemi, F., Rahvar, S., and Tabar, M. R. R.: Multifractal detrended fluctuation analysis of sunspot time series, J. Stat. Mech.-Theory E., 2, doi:10.1088/17425468/2006/02/P02003, 2006.

Peng, C. K., Buldyrev, S. V., Havlin, S., Simons, M., Stanley, H. E., and Goldberger, A. L.: Mosaic organization of DNA nucleotides, Phys. Rev. E, 49, 1685-1689, 1994.

Pulkkinen, A., Klimas, A., Vassiliadis, D., Uritsky, V., and Tanskanen, E.: Spatiotemporal scaling properties of the gound geomegnetic field variations, J. Geophys. Res., 111, A03305, doi:10.1029/2005JA011294, 2005.

Taqqu, M. S., Teverovsky, V., and Willinger, W.: Estimators for long-range dependence: an empirical study, Fractals, 3, 785788, 1995.

Wanliss, J. A.: Nonlinear variability of SYM-H over two solar cycles, Earth Planets Space, 56, e13-16, 2004.

Wanliss, J. A.: Fractal properties of SYM-H during quiet and active times, J. Geophys. Res., 110, A03202, doi:10.1029/2004JA010544, 2005.

Wanliss, J. A., Anh, V. V., Yu, Z. G., and Watson, S.: Multifractal modelling of magnetic storms via symbolic dynamics analysis, J. Geophys. Res., 110, A08214, doi:10.1029/2004JA010996, 2005.

Wanliss, J. A. and Dobias, P.: Space Storm as a Phase Transition, J. Atmos. Sol. Terr. Phys., doi:10.1016/j.jastp.2007.01.001, 69, 675-684, 2007.

Yu, Z. G., Anh, V., and Wang, B.: Correlation property of length sequences based on global structure of complete genome, Phys. Rev. E, 63, 011903, 2001.

Yu, Z. G., Anh, V. V., Lau, K. S., and Zhou, L. Q.: Fractal and multifractal analysis of hydrophobic free energies and solvent accessibilities in proteins, Phys. Rev. E, 63, 031920, 2006.

Yu, Z. G., Anh, V. V., Wanliss, J. A., and Watson, S. M.: Chaos game representation of the Dst index and prediction of geomagnetic storm events, Chaos Soliton. Fract., 31, 736-746, 2007. 